\title{
Informes finales. Conclusiones de los talleres
}

Taller 1. ¿Qué se ha hecho en el plan de igualdad de género?

Dinamizadora: María Honrubia Pérez (profesora de Enfermería, Universidad de Barcelona)

El taller dio comienzo con una exposición por parte de la delegada del Rector y presidenta de la Comisión para la Igualdad de Género de la Universidad de Barcelona, Dra. María Antonia Pujol, en la que contextualizó la trayectoria de la Comisión desde su constitución en el año 2007; destacó las acciones más relevantes realizadas como la elaboración del I Plan de Igualdad, la constitución de comisiones de Igualdad de Género con el nombramiento de las presidencias de las mismas en las diferentes facultades y centros de la Universidad de Barcelona, la celebración en marzo de 2012 del I Encuentro sobre Género de las Universidades Españolas, la elaboración del II Plan de Igualdad y su publicación en la web de la Comisión de la Universidad de Barcelona.

Las actividades en vigor en estos momentos son trabajar en los 10 ejes del II Plan de Igualdad que se han distribuido entre las diferentes comisiones de las facultades para su desarrollo y puesta en práctica y la elaboración de un protocolo único de intervención sobre violencia de género para la Universidad de Barcelona, a partir de los tres existentes, en las facultades de Derecho y Económicas respectivamente, aprobados en sus juntas de Facultad y un tercero a punto de finalizar su redacción, en la Facultad de Bellas Artes.

A continuación, tomaron la palabra las diferentes comisiones de las facultades y centros de Ciencias de la Salud: la Dra. M. ${ }^{\text {a }}$ Victoria Carreras de la Facultad de Psicología, la Dra. Amalia Lafuente de la Facultad de Medicina Campus Pi i Suñer, las Dras. Silvia Sánchez de la Facultad de Odontología y M. ${ }^{a}$ Carmen Olivé de la Escuela de Enfermería del Campus de Bellvitge y el Dr. Francesc Guardiola de la Facultad de Farmacia. Explicaron su nombramiento a la presi- dencia de las mismas y describieron las acciones desarrolladas en estos años: actividades dirigidas a visibilizar a mujeres relevantes en sus respectivas disciplinas, conferencias, cineforum, exposiciones, actos conmemorativos los días 8 de marzo y 25 de noviembre, estudios sobre las diferencias de género en las escalas del profesorado universitario y del estudiantado, actividades formativas en el aula, propuestas de establecer convenios con instituciones externas a la UB, como el Instituto Catalán de las Mujeres y abrir nuevas vías de colaboración con otras instituciones dentro de la UB como el Instituto de Ciencias de la Educación (ICE) y el Centro de Recursos para el Aprendizaje y la Investigación (CRAI).

A continuación, se abrió un debate con la cuestión ¿que hacer a partir de ahora? Fruto de este debate, se generan unas conclusiones:

1. Se reconoce la necesidad de tener una normativa escrita con las actuaciones que deben llevar a cabo los centros cuando se produzcan actos de acoso entre el estudiantado.

2. Se adquiere el compromiso por parte de la delegada del Rector de visualizar a través de su web todas las actividades que se realicen sobre género en la Universidad de Barcelona.

3. Visibilizar las comisiones de Igualdad de Ciencias de la Salud, introduciendo la información de quienes las componen y de sus actividades en las webs de las respectivas facultades y centros.

4. Invitar al profesorado de Ciencias de la Salud a que integre en las presentaciones sus clases, la referencia visual del Día Contra la Violencia de Género, durante la semana de noviembre en la que se celebra.

5. Enviar a la Comisión para la Igualdad de Género de la Universidad de Barcelona todas las actividades que se realicen en cada centro de Ciencias de la Salud con la finalidad de tener constancia centralizada y facilitar su difusión a toda la comunidad universitaria. 
6. Establecer mecanismos de comunicación fluida entre las diferentes comisiones de Igualdad de los centros de Ciencias de la Salud.

7. Desarrollar desde las comisiones de cada centro de Ciencias de la Salud los ejes del II Plan de Igualdad encomendados por la Comisión para la Igualdad de Género de la Universidad de Barcelona.

8. Conseguir una mayor cohesión entre las comisiones de Igualdad de las facultades y centros de Ciencias de la Salud.

Las presentaciones realizadas por las diferentes comisiones de los centros, durante el taller, se encontrarán expuestas en el repositorio de la Universidad.

Taller 2. Innovación docente en Ciencias de la Salud: Trabajos de innovación

Dinamizadoras: Mercedes Gracenea Zugarramurdi (secretaria de ICE - UB), María Honrubia Pérez (profesora de Enfermería - UB)
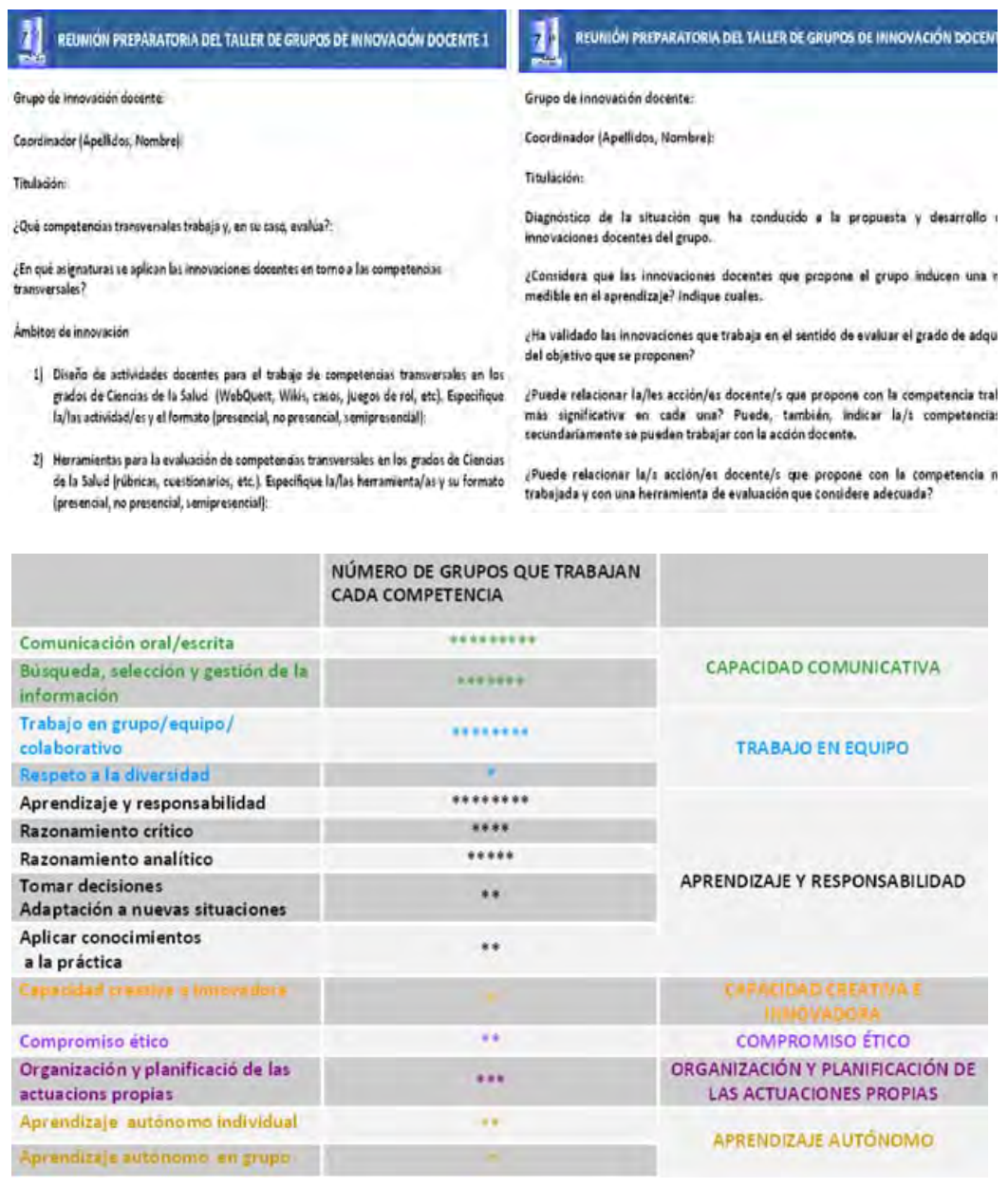

"Grupo que trabaja la competencia.
La Universidad de Barcelona propone seis competencias transversales que deben adquirir los estudiantes de sus titulaciones y los documentos Verifica de esas titulaciones adscriben las competencias transversales a las materias básicas específicas y optativas que conforman los grados. No obstante, los Verifica no determinan la estrategia docente que se debe seguir y queda a cargo de los grados el diseño de las acciones docentes que implementen para favorecer el desarrollo de las competencias por parte de los estudiantes y su evaluación y acreditación.

En este contexto, el 7. ${ }^{\circ}$ Encuentro de Profesorado de Ciencias de la Salud propone organizar un taller colaborativo dirigido a los grupos de innovación docente consolidados (GIDc) de la Universidad de Barcelona con el objetivo de recoger las acciones docentes propuestas y desarrolladas por estos grupos para la adquisición de las competencias transversales en los grados y constituir un foro de debate de las líneas de innovación docente a seguir en esta área.

Participan 20 GIDc del ámbito de las Ciencias de la Salud, con actividad en los grados de Ciencias Biomédicas (1), Farmacia (9, e-Galénica, GID en Análisis Instrumental Farmacéutico, Orfila, Tecnología Farmacéutica, Grupo de Alternativas Metodológicas en Fisiología y Fisiopatología, GID en Farmacología, Unidad de Laboratorios Docentes, Enseñar a aprender Fisiología, Unidad de Farmacia Clínica y Farmacoterapia), Enfermería (1, Grupo de Enfermería Orientado a Técnicas Educativas Innovadoras), Medicina (2, Instrucción y Aprendizaje en Ciencias de la Salud, GID de Anatomía Virtual y de Simulación en Medicina), Nutrición Humana y Dietética (1, Alternativas Metodológicas en Fisiología y Fisiopatología) y Psicología (6, GID en Psicología del Desarrollo, GID para la Mejora de la Docencia de Diseños Técnicos y de Investigación Psicológica y de la Salud, GID en Psicología Social, Grupo para el desarrollo para el aprendizaje de la estadística en salud y GID en Comunicación Audiovisual).

Los GIDc aportan, previamente al taller, los datos referentes a su actividad respondiendo dos fichas consensuadas en reuniones preparatorias (Figura).

Los grupos trabajan las competencias transversales Capacidad comunicativa, Trabajo en equipo, Capacidad de aprendizaje y responsabilidad, Capacidad creativa e innovadora y Compromiso ético. Adicionalmente, consideran otras capacidades como Organización y planificación de las actuaciones propias y Aprendizaje autónomo tanto individual como en grupo. La competencia transversal 
que más interés ha suscitado ha sido la Capacidad comunicativa, que trabajan 16 grupos, tanto en su dimensión de Comunicación oral y escrita (nueve grupos) como en la de Búsqueda, selección y gestión de la información (siete grupos). El trabajo en equipo ocupa a nueve grupos, ocho de los cuales actúan en Trabajo en grupo/equipo/colaborativo y uno en Respeto a la diversidad. La Capacidad de aprendizaje y responsabilidad la tratan la práctica totalidad de grupos, bien como tal o en sus diversas dimensiones de Razonamiento crítico, Razonamiento analítico, Capacidad de tomar decisiones, Capacidad de adaptarse a nuevas situaciones y Capacidad de aplicar los conocimientos a la práctica. Por el contrario, la Capacidad creativa e innovadora la trabaja únicamente un grupo y el Compromiso ético, dos grupos. Cabe destacar que la Sostenibilidad no está presente en las líneas de trabajo de los grupos.

Los GIDc han diseñado varios tipos de acciones docentes para el desarrollo de las competencias transversales, como webquests, resolución de casos, problemas, ejercicios y cuestiones, análisis de textos científicos, análisis y evaluación de datos, interpretación de imágenes en $3 \mathrm{D}$, web conferencias, páginas web, clikers, wikis, entre otros, que utilizan para el desarrollo de más de una competencia.

En este contexto, los grupos consensuaron dos reflexiones:

- Intensificar el trabajo de las competencias transversales menos presentes en los grupos como la Sostenibilidad, el Compromiso ético y la Capacidad creativa e innovadora, de manera colaborativa y consensuada, transversalmente en los grados de Ciencias de la Salud.

- Consensuar acciones docentes y actividades evaluatorias para las competencias que se trabajan intensivamente, como la Capacidad de aprendizaje y responsabilidad, la Capacidad comunicativa y el Trabajo en equipo, con la finalidad de llegar a propuestas unitarias para el trabajo de estas competencias.

Los GIDc consideran oportuno realizar una publicación conjunta sobre el estado actual de trabajo de las competencias transversales en Ciencias de la Salud que refleje el cumplimiento de los encargos de los documentos Verifica de los grados y recoja los datos aportados al taller. Asimismo, han reflexionado sobre la pertinencia de constituir una red de grupos de Ciencias de la Salud para avanzar conjuntamente en el trabajo de las competencias transversales en este ámbito.

\section{Taller 3. La semipresencialidad}

Dinamizadores: Joan Simon Pallisé (GIBAF de la UB), Mónica Arenas Ortigosa (CRAI Fac. Farmacia UB), Silvia Argudo, Josefina Blasco, Ramon Gabara

En el marco del 7..$^{\circ}$ Encuentro de Profesorado de Ciencias de la Salud, ha tenido lugar el taller sobre semipresencialidad bajo el título de La semipresencialidad: buenas prácticas docentes, con la participación de un total de 25 colegas. Este taller, coordinado por el profesor Joan Simon (Facultad de Farmacia) y por Mónica Arenas (CRAI Facultad de Farmacia), también ha contado como ponentes en la mesa redonda con las aportaciones de las profesoras Josefina Blasco (Facultad de Biología), Silvia Argudo y el estudiante Ramón Gabara, ambos de la Facultad de Biblioteconomía y Documentación.

El trabajo se ha llevado a cabo en torno a cuatro grandes ámbitos con fuerte incidencia en la docencia semipresencial: 1) Conceptualización; 2) Personas (perfil del estudiantado y perfil del profesorado); 3) Metodología docente, y 4) Formación.

Desde el punto de vista organizativo, el taller ha tenido un marcado carácter participativo a través del debate en pequeño grupo y posterior discusión conjunta con los compañeros que tienen experiencia en los diferentes ámbitos propuestos. La encuesta de opinión indica un alto grado de satisfacción tanto por la propia mecánica del debate como del taller en su globalidad. De este debate destacamos las conclusiones más relevantes de cara a futuras nuevas implementaciones de esta modalidad semipresencial en nuestra universidad:

1. La característica que delimita mejor el concepto de semipresencialidad, a la vez que permite una clara diferenciación con la presencialidad con el uso intensivo de las tecnologías, es la metodología docente. En ningún caso se puede caer en la trampa de pensar que son cuestiones tecnológicas y de utilización de herramientas y espacios virtuales las que dan carácter a la modalidad semipresencial. Por lo tanto, es absolutamente necesario un diseño metodológico específico para aprovechar el potencial de la semipresencialidad. Las prácticas clínicas, de laboratorio o de campo son uno de los principales impedimentos a la hora de plantearse esta modalidad.

2. La modalidad semipresencial puede estar especialmente indicada para estudiantes ya integrados en el mundo laboral. También, pero en menor medida, puede ir dirigida a perfiles de estudiantes repetidores o estudiantes con alto rendimiento académico que deseen una doble titulación. En cualquier caso, se requiere de estos 
estudiantes una alta responsabilidad frente a su propio aprendizaje además de una buena gestión del propio tiempo.

3. El profesorado encargado de impartir esta modalidad no tiene por qué ser altamente tecnófilo con respecto al dominio de diferentes herramientas informáticas. Sin embargo, son deseables equipos docentes cohesionados con profesorado novel y sénior, y siempre con destacadas habilidades comunicativas en red.

4. El cambio metodológico imprescindible para la impartición de docencia semipresencial de calidad implica, de entrada, el abandono de las clases magistrales puramente expositivas frente a las clases de resolución de dudas y de reflexión/razonamiento compartido. Los materiales y recursos deben tener una alta disponibilidad. Entre las actividades que se asocian en esta modalidad cabe destacar los foros, los cuestionarios autoevaluativos y los wikis, sin rehuir, sin embargo, otras herramientas y servicios externos al Campus Virtual. Estas actividades deben tener un ca- rácter más formativo que exclusivamente acreditativo y siempre dentro de un marco de evaluación continua. La tutorización y seguimiento cercano del estudiante de esta modalidad es del todo esencial para evitar el fracaso académico.

5. La formación del profesorado es básica y primordial para comenzar con esta nueva modalidad, si se quiere exigir calidad. A pesar de que se dispondrá de suficiente formación en habilidades instrumentales relacionadas con la tecnología y la red, no se puede olvidar la conveniencia de disponer de formación también en aspectos didácticos y pedagógicos en el uso de estas herramientas y, también, en técnicas de gestión del tiempo y tutorización y seguimiento del estudiante. Deficiencias en esta formación puede conllevar un alto grado de improvisación en las actividades a través del Campus Virtual. Es fundamental disponer de un plan de formación bien estructurado para llevar a cabo metódicamente la formación del profesorado y para garantizar la excelencia, también, en la modalidad semipresencial. 